\title{
一种红光发射三苯胺吡啶盐的合成及荧光探针性质
}

\author{
陶在琴 钱 鹰*
}

(东南大学化学化工学院 南京 211189)

\begin{abstract}
摘要 以双碘代芳醛 4-[N,N-二(4-碘苯基)氨基]苯甲醛与 4-乙烯基吡啶通过钯催化双位点 Heck 偶联反应制备了中间体 TPAPy, TPAPy 再与碘代十八烷反应得到吡啶盐衍生物 TPAPyS. 目标化合物的结构经过红外光谱、核磁共振谱、高分 辨质谱确认, 测定了吡啶盐 TPAPyS 在固态、水溶液中及乙醇/水混合溶液中的苂光光谱. 吡啶盐 TPAPyS 在固体状态 下发出暗红色荧光, 苂光发射峰为 $654 \mathrm{~nm}$, 测得固体量子产率为 $3.83 \%$. TPAPyS 在水溶液中发出红色荧光, 菼光发射 峰为 $647 \mathrm{~nm}$. 在乙醇/水混合溶液中, 化合物 TPAPyS 的苂光发射峰位于 $612 \sim 640 \mathrm{~nm}$. 测定了吡啶盐 TPAPyS 与牛血 清蛋白(BSA)、胱氨酸(DCys) 及半胱氨酸(Cys)在生理条件下的光谱行为, 吡啶盐 TPAPyS 与 BSA、氨基酸作用后苂光 发射强度均增加. 吡啶盐 TPAPyS 是一种可溶于水的红光发射材料, 荧光发射峰位于近红外波段, 可作为荧光探针用 于牛血清蛋白和氨基酸的检测.
\end{abstract}

关键词 水溶性吡啶盐; 苂光探针; Heck 反应; 红光发射; 苂光性质

\section{Systhesis and Fluorescence Probe Properities of Red-Emission Triphenylamine-Pyridinium Salt}

\author{
Tao, Zaiqin Qian, Ying* \\ (School of Chemistry and Chemical Engineering, Southeast University, Nanjing 211189)
}

\begin{abstract}
Intermediate TPAPy was synthesised by vinyl-pyridine and 4-[N,N-bis(4-iodophenyl)amino]benzaldehyde through double-pot Heck reaction, then iodooctadecane was introduced to pyridyl forming the final pyridine salt TPAPyS. The structure of final salt was characterized by ${ }^{1} \mathrm{H}$ NMR, ${ }^{13} \mathrm{C}$ NMR, IR and HRMS-MALDI-TOF. Its fluorescence property was examined. Pyridine salt TPAPyS emits dark red fluorescence with peaks at $654 \mathrm{~nm}$ in solid state. The solid-state quantum yield of TPAPyS is $3.83 \%$. TPAPyS emits red fluorescence with peaks at $647 \mathrm{~nm}$ in pure water and $612 \sim 640 \mathrm{~nm}$ in ethanol $/ \mathrm{H}_{2} \mathrm{O}$ mixture solution. The spectra of TPAPyS with or without BSA, dicysteine, cysteine were measured. The maxima fluorescence emission intensity of water-soluble pyridine salt TPAPyS is enhanced after combining with BSA, dicysteine and cysteine. Pyridine salt TPAPyS is water-soluble with fluorescence spectra in near-infrared region, and it can be used as fluorescence probe to detect BSA, DCys and Cys.
\end{abstract}

Keywords water-soluble pyridine salt; fluorescence probe; Heck reaction; red emission; fluorescence properity

近些年来, 红光发射材料由于其在光纤通讯、激光 系统、近红外增感、离子检测 ${ }^{[1 \sim 5]}$ 、细胞荧光成像 ${ }^{[6 ~ 15] 、}$ 荧光探针 ${ }^{16-20]}$ 等方面的广泛应用而引起科学家的关注. 目前, 已经报道的红光发射材料除金属离子掺杂的化合 物、金属络合物、稀土配合物外，还有一类结构稳定、 光学性能良好的吡啶盐红光发射材料, 这类材料的红光 发射对生物组织伤害小, 可用于生物苂光探针的研究. 2006 年, $\mathrm{Xu}$ 等 ${ }^{[21]}$ 报道了一种红光发射吡啶盐材料, 并将 其用于单层电致发光器件, 获得了稳定的红色电致发
光. 2007 年, Jin 等 ${ }^{[22]}$ 报道了一种水溶性甲基吡啶衍生物, 其在水溶液中的荧光发射峰达到了 $627 \mathrm{~nm}$, 将其用于 ctDNA 检测, 发现 ctDNA 在 $0 \sim 50 \mu \mathrm{g} / \mathrm{mL}$ 范围内, 荧光 强度变化与 ctDNA 浓度呈良好的线性关系, $K_{\mathrm{sv}}$ 常数达 到 $1.4 \times 102.2009$ 年, Elacqua 等 ${ }^{[23]}$ 在吡啶基的 $\mathrm{N}$ 原子上 引入甲基、乙基形成两种吡啶盐衍生物, 发现烷基的引 入能够使分子的荧光发射峰红移. 2013 年，一系列单、 双、三乙烯基吡啶三苯胺盐被报道出来，它们的发射波 长在 $590 \sim 680 \mathrm{~nm}$, 有着良好的双光子吸收性质，被用

* E-mail: yingqian@seu.edu.cn

Received March 30, 2014; revised May 26, 2014; published online July 11, 2014.

Project supported by the National Natural Science Foundation of China (No. 61178057).

国家自然科学基金(No.61178057)资助项目 
于 DNA 探针研究以及苂光细胞成像 ${ }^{[24]}$.

本文报道了一种可溶于水的三苯胺吡啶盐 TPAPyS, 结构式如图 1 所示. TPAPyS 在固态及水溶液中均发出红 色苂光, 波长范围在 $640 \sim 660 \mathrm{~nm}$ 的近红外区域. 测定 了阳离子吡啶盐 TPAPyS 的固体苂光、固体量子效率、 在有机溶剂中的苂光、在纯水中的苂光、在乙醇/ $\mathrm{H}_{2} \mathrm{O}$ 混合溶剂中的聚集态荧光. 研究了吡啶盐 TPAPyS 与牛 血清蛋白(BSA)及氨基酸在生理条件下相互作用的苂光 光谱, 测定了 TPAPyS 与 BSA 作用的圆二色谱, 并计算 了 TPAPyS 与 BSA 的结合常数 $K_{\mathrm{sv}}$ 以及 BSA 的 $\alpha$-螺旋 含量.

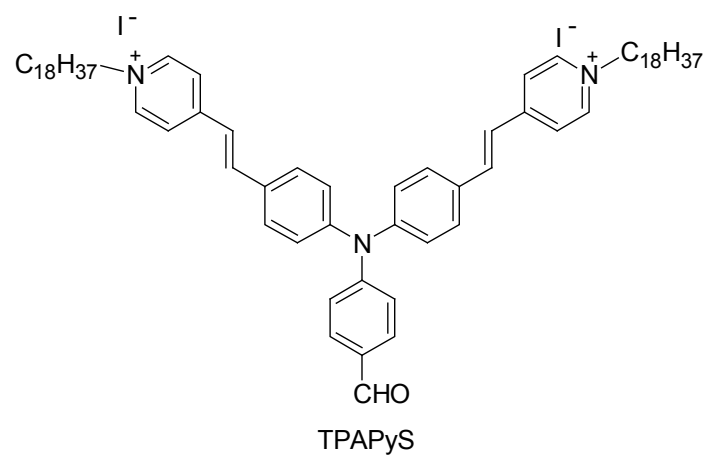

图 1 三苯胺吡啶盐 TPAPyS 的结构图

Figure 1 The structure of triphenylamine pyridium TPAPyS

\section{1 结果与讨论}

本文用双碘代三苯胺衍生物和 4-乙烯基吡啶通过 Pd 催化 Heck 反应得到三苯胺吡啶衍生物 TPAPy, 再与 碘代十八烷缩合, 得到可溶水、红光发射的三苯胺吡啶 盐衍生物 TPAPyS. 讨论了化合物 TPAPyS 的固体荧光、 在水溶液中的苂光、在有机溶剂中的苂光. 研究了 TPAPyS 与牛血清蛋白、氨基酸相互作用的苂光光谱.

\section{1 三苯胺吡啶盐 TPAPyS 的合成方法}

Pd 催化下的 Heck 偶联反应是合成碳碳双键的有 效方法之一 ${ }^{[25]}$. 由双碘代芳基醛和 4-乙烯基吡啶通过 Pd 催化下的 Heck 偶联反应合成了的三苯胺吡啶衍生物 TPAPy, 然后在生色团 TPAPy 的两个吡啶基上分别引入 十八烷基柔性链, 得到水溶性三苯胺吡啶盐衍生物 TPAPyS, 产物经过红外光谱、核磁共振谱、高分辨质谱 鉴定, 合成路线如图 2 所示.

\section{2 三苯胺吡啶盐的红光发射}

测定了三苯胺吡啶盐 TPAPyS 的固体荧光光谱, 如 图 3 所示, TPAPyS 固体粉末在 $430 \mathrm{~nm}$ 紫外光激发下, 苂 光发射峰为 $654 \mathrm{~nm}$, 位于近红外波段, 实验测其固体苂 光量子效率为 $3.83 \%$.<smiles>O=Cc1ccc(N(c2ccc(I)cc2)c2ccc(I)cc2)cc1</smiles>
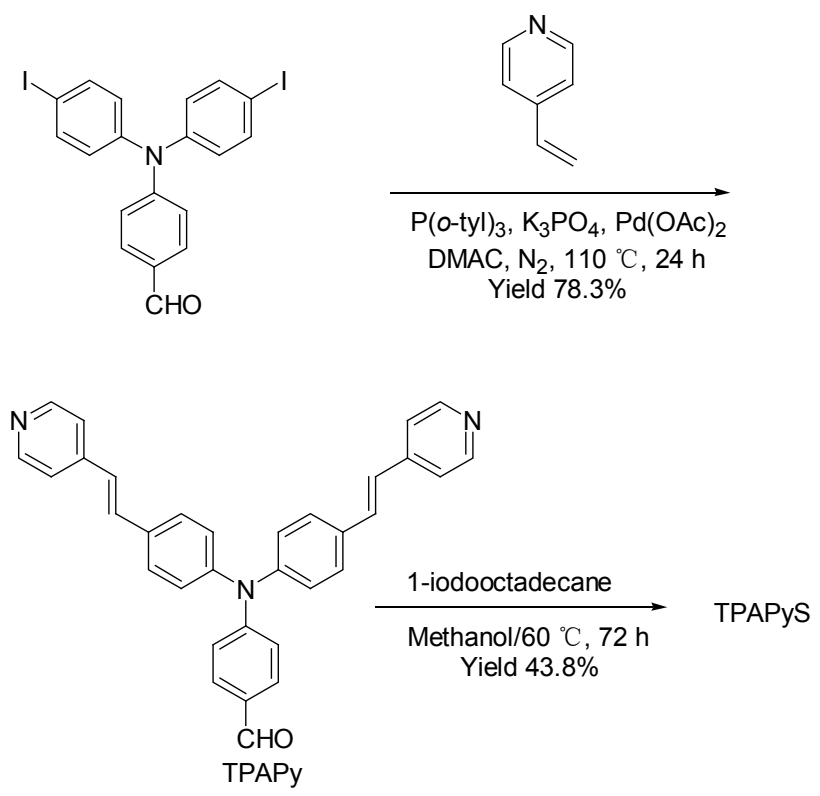

图 2 三苯胺吡啶衍生物 TPAPy 和 TPAPyS 的合成路线 Figure 2 The synthetic route of triphenylamine pyridine derivatives TPAPy and TPAPyS

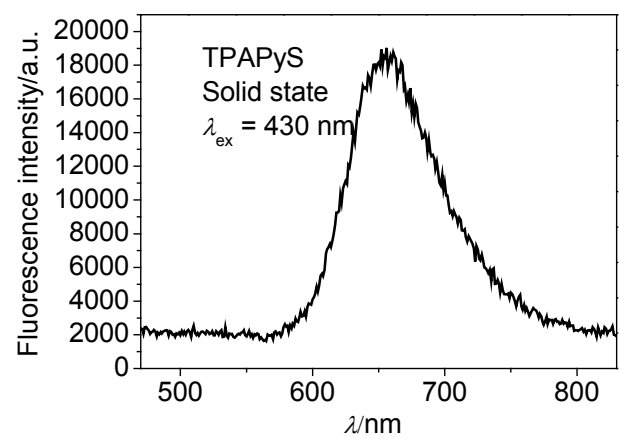

图 3 化合物 TPAPyS 的固体荧光发射图谱

Figure 3 Solid fluorescence emission spectra of compound TPAPyS

文献报道的大部分荧光探针不溶于水，而三苯胺吡 啶盐 TPAPyS 可溶于水, 实验测定了吡啶盐 TPAPyS $\left(1.0 \times 10^{-6} \mathrm{~mol} / \mathrm{L}\right)$ 在纯水中的紫外-苂光性质，如图 4 所 示. 由图 4 可知吡啶盐 TPAPyS 在水中的苂光发射峰为 $647 \mathrm{~nm}$, 属近红外红光发射. TPAPyS 在纯水中有两个吸 收带, 分别为 320 和 $474 \mathrm{~nm}$, 其中最大吸收峰为分子内 电荷转移态的吸收, 对应 $\pi-\pi *$ 跃迁吸收. 紫外吸收光谱 与苂光发射光谱呈很好的镜像关系.

TPAPyS 在有机溶剂中有着良好的溶解性, 在水中 也有一定的溶解性. 实验以最大紫外吸收处波长作为激 发波长，测定了 TPAPyS 在不同比例的乙醇/ $\mathrm{H}_{2} \mathrm{O}$ 混合体 系中的菼光光谱. 图 5 为 TPAPyS 在乙醇/ $\mathrm{H}_{2} \mathrm{O}$ 体系中水 含量为 $0 \%\left(\lambda_{\mathrm{ex}}=459 \mathrm{~nm}\right), 10 \%\left(\lambda_{\mathrm{ex}}=444 \mathrm{~nm}\right), 60 \%\left(\lambda_{\mathrm{ex}}=\right.$ $445 \mathrm{~nm}), 100 \%\left(\lambda_{\mathrm{ex}}=451 \mathrm{~nm}\right)$ 时的苂光光谱. 由图 5 可 


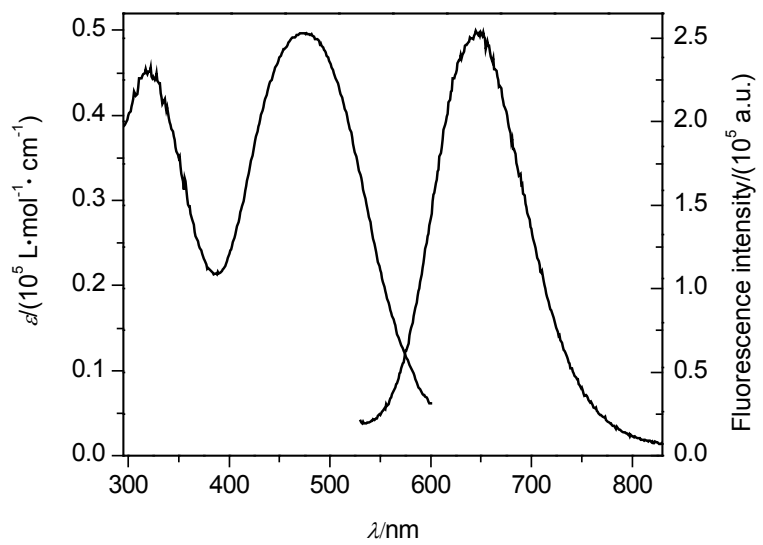

图 4 水溶性吡啶盐 TPAPyS 在纯水中 $\left(1.0 \times 10^{-6} \mathrm{~mol} / \mathrm{L}\right)$ 的紫 外吸收-苂光发射图

Figure 4 UV absorption and single-photon fluorescence spectra of compound TPAPyS in pure water $\left(1.0 \times 10^{-6} \mathrm{~mol} / \mathrm{L}\right)$

知, 在混合体系中, 当水含量由 $0 \%$ 增加到 $10 \%$ 时, 溶液 的荧光发射峰由 $619 \mathrm{~nm}$ 蓝移到 $614 \mathrm{~nm}$. 荧光发射强度 降低; 当水含量由 $10 \%$ 增加到 $60 \%$ 时，苂光发射峰没有 明显变化, 溶液的荧光发射强度降低到原来的 0.61 倍; 当水含量由 $60 \%$ 增加到 $100 \%$ 时, 苂光发射峰由 $612 \mathrm{~nm}$ 红移到 $640 \mathrm{~nm}$, 苂光发射强度大大降低, 但并没有完全 淬灭. 整个过程中, 吡定盐 TPAPyS 由溶液状态变成纳 米聚集态, 随着水含量的增加, 混合体系溶剂极性增大, 使得荧光发射强度减弱, 最大荧光发射波长先略微蓝移 最终红移.

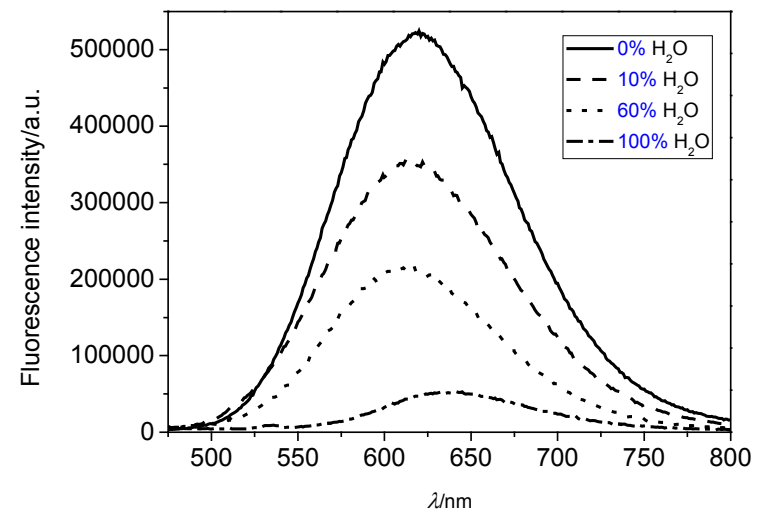

图 5 吡啶盐 TPAPyS $\left(1.0 \times 10^{-4} \mathrm{~mol} / \mathrm{L}\right)$ 在水含量为 $0 \%, 10 \%$, $60 \%, 100 \%$ 的乙醇 $/ \mathrm{H}_{2} \mathrm{O}$ 体系中的荧光发射图

Figure 5 Fluorescence spectra of $1.0 \times 10^{-4} \mathrm{~mol} / \mathrm{L}$ TPAPyS in the ethanol $/ \mathrm{H}_{2} \mathrm{O}$ mixture at different water fraction

具有聚集态荧光的吡啶盐类化合物少有报道 ${ }^{[22,26]}$, 而吡啶盐 TPAPyS 就是一种有聚集态荧光的化合物, 这 是由于吡啶盐 TPAPyS 自身结构刚性化导致分子内旋转 受限, 产生聚集态荧光.
三苯胺吡啶盐 TPAPyS 在有机溶剂中有很好的溶解 性. 图 6 为中间体 TPAPy 和 TPAPyS 在 $\mathrm{CH}_{2} \mathrm{Cl}_{2}$ 溶液中 $\left(1.0 \times 10^{-5} \mathrm{~mol} / \mathrm{L}\right)$ 的紫外吸收-荧光发射归一化图. 由图 6 可知 TPAPy 的荧光发射峰为 $528 \mathrm{~nm}$, 而 TPAPyS 的荧 光发射峰为 $621 \mathrm{~nm}$, 红移了 $93 \mathrm{~nm}$. TPAPy 和 TPAPyS 在 $\mathrm{CH}_{2} \mathrm{Cl}_{2}$ 溶液中均有两个吸收带, TPAPy 的吸收峰分别 为 287 和 $380 \mathrm{~nm}$, TPAPyS 的吸收峰分别为 334 和 495 $\mathrm{nm}$, 由此可见成盐后紫外吸收峰也大幅度红移了. 由于 吡啶盐的拉电子能力大于成盐前, TPAPyS 具有较强的 分子内电荷转移, 所以化合物 TPAPyS 相对于 TPAPy 在 有机溶剂中的紫外吸收峰和荧光发射峰波长均有红移.

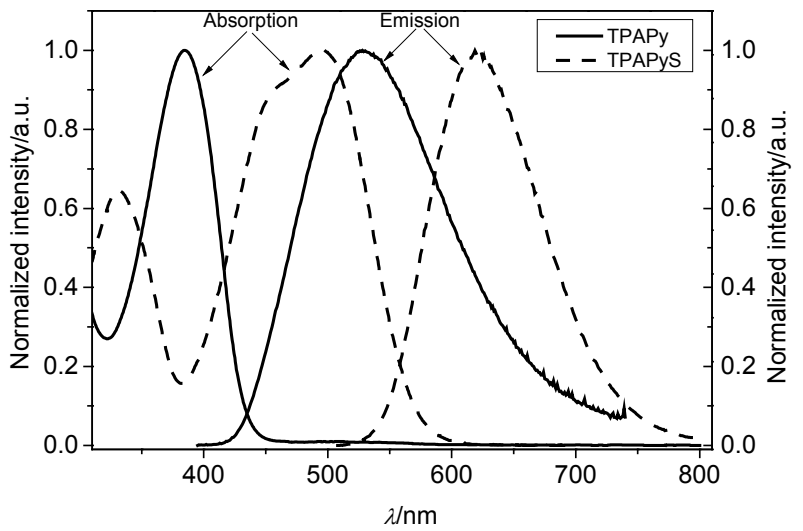

图 6 化合物 TPAPy 和 TPAPyS 在 $\mathrm{CH}_{2} \mathrm{Cl}_{2}$ 溶液中归一化的紫 外吸收一苂光发射光谱

Figure 6 Normalized UV absorption spectra and single-photon fluorescence spectra of compounds TPAPy and TPAPyS in $\mathrm{CH}_{2} \mathrm{Cl}_{2}$

本文以最大紫外吸收处的波长作为激发波长, 测定 了吡啶盐化合物 TPAPyS 在甲苯 $\left(\lambda_{\mathrm{ex}}=453 \mathrm{~nm}\right)$ 、四氢呋 喃(THF) $\left(\lambda_{\mathrm{ex}}=464 \mathrm{~nm}\right)$ 、乙醇 $\left(\lambda_{\mathrm{ex}}=459 \mathrm{~nm}\right) 、 \mathrm{CH}_{2} \mathrm{Cl}_{2}$ $\left(\lambda_{\mathrm{ex}}=493 \mathrm{~nm}\right) 、 N, N$-二甲基甲酰胺(DMF) $\left(\lambda_{\mathrm{ex}}=445 \mathrm{~nm}\right)$ 中的苂光光谱, 如图 7 所示. 由图 7 可以看出, TPAPyS 在 5 种溶剂中的最大荧光发射峰分别为 612(甲苯), 615 (THF), 620(乙醇), $621\left(\mathrm{CH}_{2} \mathrm{Cl}_{2}\right), 627$ (DMF) $\mathrm{nm}$, 吡啶盐 的荧光发射强度基本上是随着溶剂极性的增大而减小 的，荧光发射峰随着溶剂极性增大而红移. 这是因为分 子吸收一定能量后，不仅具有平面的分子内电荷转移 (ICT)态, 还出现了扭转的分子内电荷转移(TICT)态, 具 有很大的极性. 此时分子就比较容易受溶剂极性的影 响. 当溶剂极性增大时, 比较利于 TICT 态的稳定, 不利 于 ICT 态的稳定, 所以分子倾向于形成 TICT 态, 导致 分子在回落的时候荧光发射强度会降低伴随着发射峰 红移. 化合物 TPAPyS 在疏水环境中(低极性)发出很强 的荧光且发射峰蓝移，由此可以将其作为荧光探针来检 测含有疏水空腔的血清蛋白. 


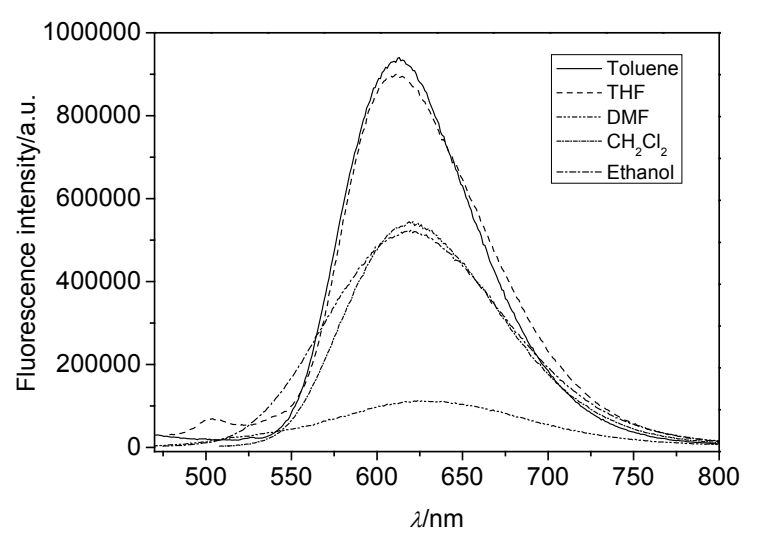

图 7 化合物 TPAPyS 在甲苯、THF、乙醇、 $\mathrm{CH}_{2} \mathrm{Cl}_{2} 、 \mathrm{DMF}$ 中的苂光发射光谱 $\left(1.0 \times 10^{-4} \mathrm{~mol} / \mathrm{L}\right)$

Figure 7 Fluorescence spectra of $1.0 \times 10^{-4} \mathrm{~mol} / \mathrm{L}$ TPAPyS in the toluene, THF, ethanol, $\mathrm{CH}_{2} \mathrm{Cl}_{2}$ and DMF

\section{3 吡啶盐与牛血清蛋白(BSA)相互作用的荧光光谱}

牛血清蛋白与人血清蛋白(HSA)具有结构同源性, 能够储存和转运众多内源性和外源性物质, 是一种非常 重要的蛋白质 ${ }^{[27 ~ 29]}$. 三苯胺吡啶盐 TPAPyS 可溶于水, 具有近红外发射的苂光特性, 可作为近红外苂光探针用 于 BSA 含量的检测. 实验中运用荧光光谱和圆二色谱 (CD)研究了 BSA 与荧光探针的作用行为 ${ }^{[30,31]}$.

在 $\mathrm{pH}$ 为 7.4 的生理环境下, 以 $\mathrm{Na}_{2} \mathrm{HPO}_{4}-\mathrm{NaH}_{2} \mathrm{PO}_{4}$ 为缓冲体系，保持探针分子 TPAPyS 浓度为 $1.0 \times 10^{-6}$ $\mathrm{mol} / \mathrm{L}, \mathrm{BSA}$ 浓度由 0 逐渐增加到 $4 \times 10^{-6} \mathrm{~mol} / \mathrm{L}$, 测得体 系的苂光发射光谱如图 8 所示. 图 8 是 $1.0 \times 10^{-6} \mathrm{~mol} / \mathrm{L}$ TPAPyS 与 $(0 \sim 4) \times 10^{-6} \mathrm{~mol} / \mathrm{L} \mathrm{BSA}$ 结合后的苂光发射 图. 由图 8 可以看出, 探针分子 TPAPyS 在与 BSA $\left(0.05 \times 10^{-6} \mathrm{~mol} / \mathrm{L}\right)$ 结合后荧光发射强度是结合前的 4 倍, 而且随着 BSA 的增加至 $4 \times 10^{-6} \mathrm{~mol} / \mathrm{L}$, 溶液的苂 光强度是结合前的 9 倍, 荧光发射峰由结合前的 $645 \mathrm{~nm}$ 蓝移到结合后的 $616 \mathrm{~nm}$, 随着 BSA 的增加继续蓝移到 $607 \mathrm{~nm}$. 由于探针 TPAPyS 在疏水性环境中会发生荧光 发射强度增加、发射峰蓝移, BSA 分子中的疏水空腔可 以很好地与探针分子发生疏水性作用, 所以当体系中 BSA 含量越来越高的时候, 疏水空腔也越来越多, 疏水 性作用越来越强, 导致荧光探针 TPAPyS 的荧光发射强 度越来越强, 而且发射峰蓝移 ${ }^{[32,33]}$.

荧光探针相对于检测物的灵敏度可以通过 Stern-Volmer 方程 ${ }^{[22]}$ 来定量研究:

$$
F / F_{0}=K_{\mathrm{sv}}[\mathrm{Q}]+1
$$

其中 $F$ 是含有给定浓度检测物的溶液的苂光强度, $F_{0}$ 是 不含检测物的溶液的荧光强度, [Q] 是检测物的浓度, $K_{\mathrm{sv}}$ 就是 Stern-Volmer 常数, 是描述检测物与 BSA 结合能力 大小的物理量.

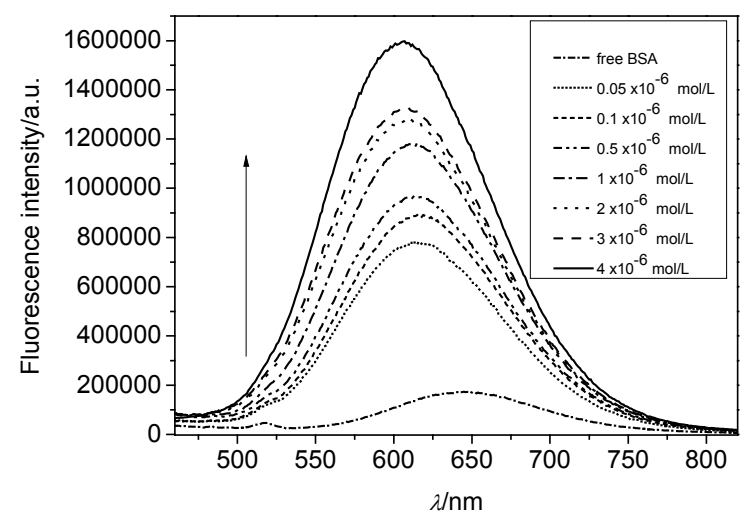

图 8 在 $\mathrm{pH}=7.4$ 的 $\mathrm{Na}_{2} \mathrm{HPO}_{4}-\mathrm{NaH}_{2} \mathrm{PO}_{4}$ 缓冲体系中探针分子 TPAPyS $\left(1.0 \times 10^{-6} \mathrm{~mol} / \mathrm{L}\right)$ 与不同浓度 BSA [ $(0 \sim 4) \times 10^{-6}$ $\mathrm{mol} / \mathrm{L}]$ 结合后的苂光发射图

Figure 8 Variation of fluorescence intensity of TPAPyS $(1.0 \times$ $\left.10^{-6} \mathrm{~mol} / \mathrm{L}\right)$ with the concentration of BSA $\left[(0 \sim 4) \times 10^{-6} \mathrm{~mol} / \mathrm{L}\right]$ in $\mathrm{Na}_{2} \mathrm{HPO}_{4}-\mathrm{NaH}_{2} \mathrm{PO}_{4}$ buffer solution

图 9 是根据图 7 计算得到的 $F / F_{0}{ }^{-1}$ 与 BSA 浓度之 间的线性关系图. 对于加入苂光探针后荧光增强的体系 来说, 其数值越大, 说明这种荧光探针越灵敏. 对于 TPAPyS, 当 BSA 浓度在 $0.05 \times 10^{-6} \sim 4.4 \times 10^{-6} \mathrm{~mol} / \mathrm{L}$ 区间时，荧光强度同 BSA 浓度之间的线性相关因子为 0.95, 呈线性关系(图 9), 这给实施检测带来很大便利. 在该线性区间，用 Stern-Volmer 计算得到的 $K_{\mathrm{sV}}$ 为 $9.39 \times 10^{5}$. 值得注意的是, 在用 TPAPyS 作苂光探针检 测 BSA 时，不仅观测到苂光增强，而且同时观测到光谱 明显蓝移了. 这意味着用 1 种苂光探针可以同时通过 2 个光谱指标对待检测物种进行分析鉴定, 相当于用 1 种 生色团实现双荧光标记, 这一性能在生物检测中具有重 要意义 ${ }^{[34 ~ 36]}$.

牛血清蛋白(BSA)在圆二色谱的远紫外区有两个明 显的负峰, 分别位于 208 和 $222 \mathrm{~nm}$, 这两个负峰都是由 于蛋白质二级结构中的肽键处于 $\alpha$-螺旋结构时肽键的 $\mathrm{n}-\pi$ * 跃迁导致的 ${ }^{[37,38]}$.

图 $10 \mathrm{~A}$ 是在模拟生理环境下, $\mathrm{pH}=7.4$ 时, 不同浓 度的 TPAPyS $\left[(0 \rightarrow 4) \times 10^{-6} \mathrm{~mol} / \mathrm{L}\right]$ 与 BSA $\left(5 \times 10^{-7}\right.$ $\mathrm{mol} / \mathrm{L}$ )结合前后的圆二色谱图. 图 10B 是根据图 $10 \mathrm{~A}$ 经 公式(2)和(3)计算得到的不同浓度 TPAPyS 与 BSA 作用 后蛋白质二级结构中的 $\alpha$-螺旋含量.

由图 10A 可以看到，探针分子是没有手性的，故而 没有负峰出现, BSA 出现了双负峰. 随着探针分子的加 入, 体系的双负峰均有变化, 峰值增加, 略微蓝移, 这 说明 BSA 中 $\alpha$-螺旋含量增加了.

BSA 与不同比例的探针分子结合前后 $\alpha$-螺旋数的 大小一般用平均残 ${ }^{[39,40]}$ 基摩尔椭圆率(mean residue ellipticity, MRE)来表示, 可以通过下面的公式计算: 


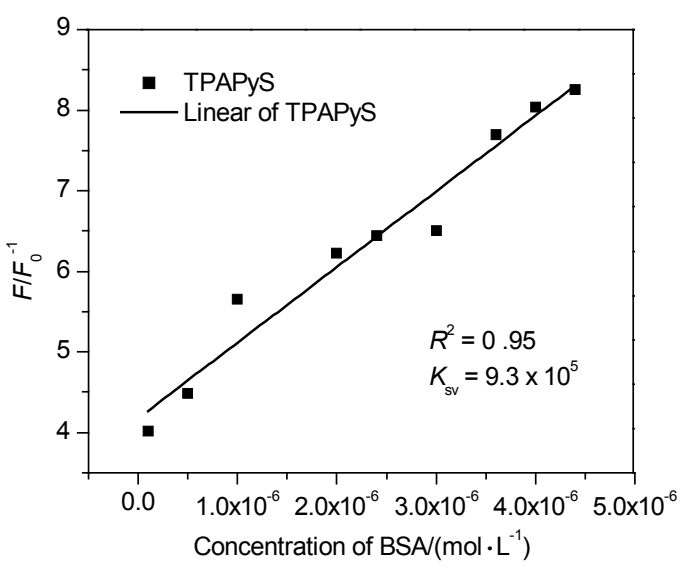

图 $9 F / F_{0}^{-1}$ 与 BSA $\left(0.05 \times 10^{-6} \rightarrow 4.4 \times 10^{-6} \mathrm{~mol} / \mathrm{L}\right)$ 浓度之间 的线性关系

Figure 9 The linear relationship between $F / F_{0}{ }^{-1}$ versus BSA concentration in the range of $0.05 \times 10^{-6} \sim 4.4 \times 10^{-6} \mathrm{~mol} / \mathrm{L}$ $c($ probe $)=1.0 \times 10^{-6} \mathrm{~mol} / \mathrm{L}$

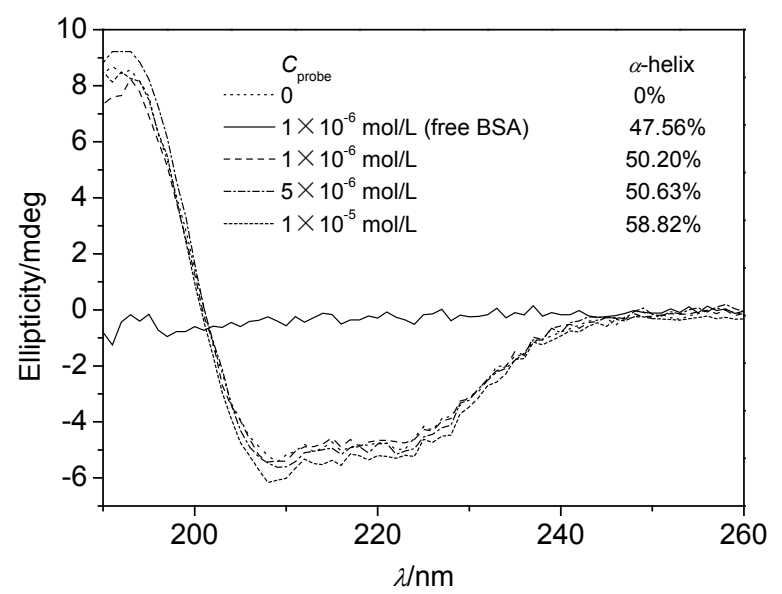

图10 不同浓度TPAPyS $\left[(0 \rightarrow 4) \times 10^{-6} \mathrm{~mol} / \mathrm{L}\right]$ 与BSA $(5 \times$

$10^{-7} \mathrm{~mol} / \mathrm{L}$ )结合前后的圆二色谱(A)及 $\alpha$-螺旋含量(B)

Figure $10 \mathrm{CD}$ spectra (A) and $\alpha$-helix (B) of BSA $\left(5 \times 10^{-7}\right.$ $\mathrm{mol} / \mathrm{L})$ with probe TPAPyS $\left[(0 \rightarrow 4) \times 10^{-6} \mathrm{~mol} / \mathrm{L}\right]$

$$
\mathrm{MRE}=\frac{\text { ObservedCD }}{C_{\mathrm{p}} n l 10}
$$

其中, $C_{\mathrm{p}}$ 是蛋白质的摩尔浓度; $n$ 是氨基酸残基数, BSA 的氨基酸残基数是 $585 ; l$ 是样品池的宽度 $\mathrm{cm}(0.1) . \alpha$-螺 旋的值可以通过 $208 \mathrm{~nm}$ 处的 MRE值得到，如方程所示：

$$
\alpha-\operatorname{helix}(\%)=\frac{-\mathrm{MRE}_{208}-4000}{33000-4000} \times 100 \%
$$

其中, $\mathrm{MRE}_{208}$ 是在 $208 \mathrm{~nm}$ 处观察到的 MRE 值; 4000 是 $208 \mathrm{~nm}$ 处无规卷曲的 MRE 值; 33000 是 $208 \mathrm{~nm}$ 处单纯 $\alpha$-螺旋的 MRE 值.

根据图 10A及公式(2), (3)可以计算出加入不同浓度 TPAPyS 后, 体系中 BSA 的 $\alpha$-螺旋含量. 图 10(B) 是不同
浓度 TPAPyS 与 BSA 作用后蛋白质二级结构中的 $\alpha$-螺 旋含量图. 由图 10B 可知, TPAPyS 与 BSA 结合后, BSA 分子二级结构中的 $\alpha$-螺旋含量增加. 苂光光谱与圆二色 谱相互印证说明与探针分子结合后蛋白质的二级构象 发生了变化 ${ }^{[30,31]}$.

总之, $\mathrm{pH}$ 为 7.4 的生理环境下, 以 $\mathrm{Na}_{2} \mathrm{HPO}_{4^{-}}$ $\mathrm{NaH}_{2} \mathrm{PO}_{4}$ 为缓冲体系, 探针分子 TPAPyS $\left(1.0 \times 10^{-6}\right.$ $\mathrm{mol} / \mathrm{L})$ 与不同浓度 BSA [(0 4.4) $\left.\times 10^{-6} \mathrm{~mol} / \mathrm{L}\right]$ 作用后, 体系的线性检测范围在 $0.05 \times 10^{-6} \sim 4.4 \times 10^{-6} \mathrm{~mol} / \mathrm{L}$ 之 间，由 Stern-Volmer 方程计算结合常数 $K_{\mathrm{sv}}$ 为 $9.3 \times 10^{5}$. 且由圆二色谱可知, BSA 与 TPAPyS 结合后蛋白质二级 结构中的 $\alpha$-螺旋含量增加. 水溶性吡啶盐 TPAPyS 可作 为近红外苂光探针用作 BSA 的检测.

\section{4 吡啶盐与氨基酸作用的荧光光谱}

氨基酸对生物体的生理平衡有着至关重要的作用. 许多氨基酸识别是利用探针的活泼基团和氨基酸的颈 基、胺基等特征基团发生反应 ${ }^{[7,41,43]}$ ，通过探针的吸收及 发射光谱的变化从而达到识别的目的. 探针分子 TPAPyS 不仅是红光发射、可溶于水，而且还有预留的 醛基，可以与不同氨基酸的氨基缩合，从而实现对氨基 酸的识别.

吡啶盐 TPAPyS 中的醛基可与 DCys, Cys 的氨基缩 合为亚胺基(图 11), 研究了吡啶盐 TPAPyS 与胱氨酸 (DCys)、半胱氨酸(Cys)相互作用的荧光光谱(图 12). TPAPyS 与 Cys 结合后荧光强度是结合前的 2 倍, 与 DCys 结合后苂光强度是结合前的 4 倍. TPAPyS 与氨基 酸作用前后荧光发射峰蓝移 $4 \mathrm{~nm}$ 左右. 图 13 是加入 DCys, Cys 前后 TPAPyS 的紫外吸收光谱. TPAPyS 与氨 基酸作用后紫外吸收均增强，蓝移 $6 \mathrm{~nm}$ 左右. 因为吡啶 盐 TPAPyS 的羰基与 DCys (Cys)的氨基发生作用, $\mathrm{C}=\mathrm{O}$ 基变成了 $\mathrm{C}=\mathrm{N}$ 基(五元杂环), 改变了吡啶盐原来的共 轭结构(图 12), 才导致了结合前后体系紫外吸收和苂光 发射光谱的变化. 水溶性吡啶盐 TPAPyS 可作为荧光探 针用作氨基酸的检测.

\section{2 结论}

通过 Heck 偶联反应合成了一种可溶于水、具有红 光发射性质的三苯胺吡啶盐衍生物 4-[N,N-二(((N-十八 烷基)吡啶碘盐-4-乙烯基)苯基)氨基]苯甲醛(TPAPyS). 吡啶盐 TPAPyS 在固体状态下发出暗红色荧光, 发射峰 为 $654 \mathrm{~nm}$, 测得固体量子产率为 $3.83 \%$. 其在纯水溶液 中的荧光发射峰为 $647 \mathrm{~nm}$, 在乙醇/水混合溶液中荧光 发射峰位于 $612 \sim 640 \mathrm{~nm}$ 之间. 测定了 $\mathrm{pH}$ 为 7.4 的 $\mathrm{Na}_{2} \mathrm{HPO}_{4}-\mathrm{NaH}_{2} \mathrm{PO}_{4}$ 缓冲体系中, 吡啶盐 TPAPyS 与牛血 清蛋白(BSA)作用的光谱行为, 测定了体积比为 $1: 9$ 的 


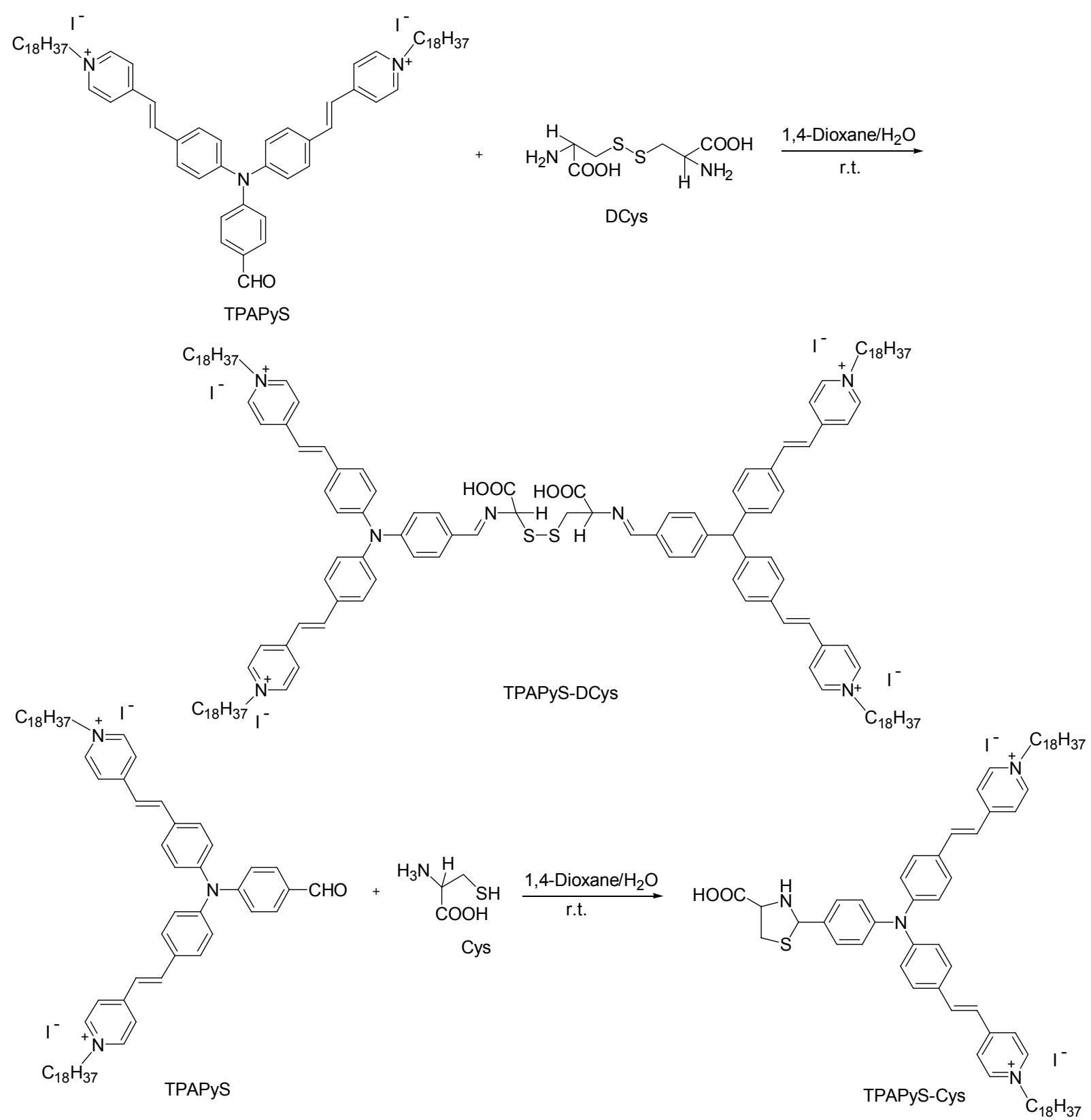

图 11 探针 TPAPyS 与胱氨酸(DCys)、半脱氨酸 (Cys) 缩合反应

Figure 11 The condensation reaction of probe TPAPyS with DCys, Cys

1,4-二氧六环/水的混合溶液中, TPAPyS 与 DCyS, Cys 结 合前后的光谱行为. 发现吡啶盐 TPAPyS 与 BSA、氨基 酸作用后苂光发射强度均增加. 吡啶盐 TPAPyS 是一种 可溶于水的红光发射材料, 苂光发射峰位于近红外波 段, 可作为荧光探针用于牛血清蛋白和氨基酸的检测.

\section{3 实验部分}

\section{1 仪器与试剂}

化合物的红外光谱用美国热电公司生产的 Nicolet 5700 型傅立叶红外光谱仪测定; 核磁共振图谱在 $\mathrm{AV}-300 \mathrm{MHz}$ 型核磁共振波谱仪(TMS 为内标, $\mathrm{CDCl}_{3}$ 为
溶剂)上测得, MALDI-TOF MS 则是用 MALDI-TOF/ TOF 和 Ultraflex II 型质谱仪测得. 熔点在 X4 型显微熔 点仪上测定; 紫外-可见吸收光谱在日本 Shimadu UV-3600 型紫外-可见分光光度计上测得; 荧光光谱是 用日本 Fluoromax-4 型苂光光谱仪测定; 圆二色谱是用 英国 Applied Photophysics 公司的 Chirascan 型号圆二色 光谱仪测得.

所有试剂均购自国药集团化学试剂有限公司. 4-乙 烯基吡啶、醋酸钯、三(邻甲基苯基)膦购自百灵威化学 股份有限公司. 碘代十八烷购自 J\&K 化学试剂公司. 胱 氨酸和半胱氨酸购自惠兴生化试剂有限公司. 所有试剂 


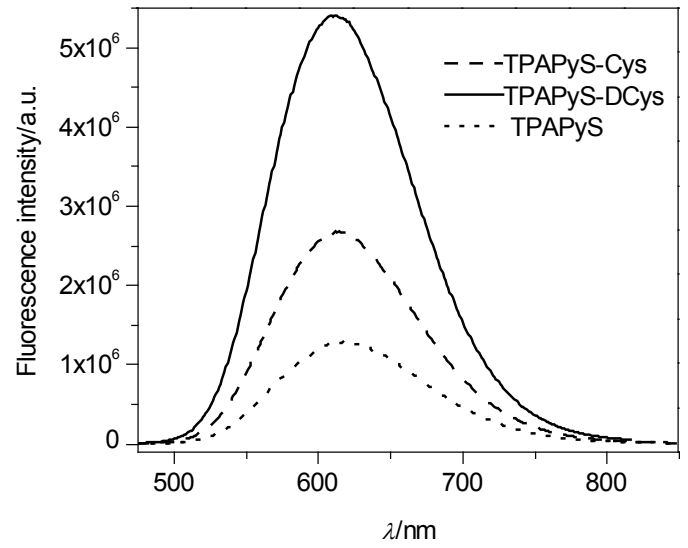

图 12 与 DCys, Cys 前后 TPAPyS 荧光发射光谱

Figure 12 Emission spectra of probe TPAPyS with or without DCys, Cys

$C($ probe $)=2 \times 10^{-5} \mathrm{~mol} / \mathrm{L}$

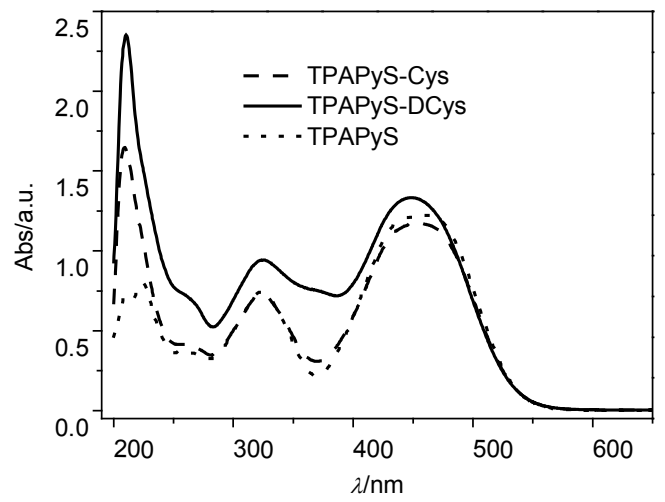

图 13 与 DCys 和 Cys 结合前后 TPAPyS 的紫外吸收光谱

Figure 13 UV spectra of probe TPAPyS with or without DCys and Cys

$$
C(\text { probe })=2 \times 10^{-5} \mathrm{~mol} / \mathrm{L}
$$

均为分析纯, 溶剂使用前用分子篎脱水处理. 4- $[N, N-$ 二 (4-碘苯基)氨基]苯甲醛参考文献[43]方法合成.

\section{2 实验方法}

3.2.14- $\{N, N-$ 二[4-(4-吡啶乙烯基)苯基]氨基 $\}$ 苯甲醛 (TPAPy) 的合成

$\mathrm{N}_{2}$ 气保护下, 分别取 4-[ $N, N$-二(4-碘苯基)氨基]苯 甲醛(M1) (0.53 g, $1.01 \mathrm{mmol})$ 和醋酸钯 $(0.023 \mathrm{~g}, 0.10$ $\mathrm{mmol})$, 三(邻甲基苯基)膦(0.061 g, $0.21 \mathrm{mmol})$, 无水磷 酸钾(0.60 g, $2.82 \mathrm{mmol})$, 4-乙烯基吡啶(1.52 mL, 13.84 $\mathrm{mmol}$ )及 $6.00 \mathrm{~mL}$ 溶剂 $\mathrm{DMF}$ 于 $25 \mathrm{~mL}$ 单口瓶中, 磁力摚 拌下, 升温至 $110{ }^{\circ} \mathrm{C}$ 反应 $24 \mathrm{~h}$. 反应结束后抽滤, 将母 液倒入 $25 \mathrm{~mL}$ 甲醇中, 有沉淀析出, 抽滤得固体并烘干, 用柱层析法提纯, 洗脱剂为 $V$ (石油醚) $: V$ (乙酸乙酯 $)=$ $8 ： 1$, 得到红色固体 $0.38 \mathrm{~g}$, 产率 $78.3 \%$. m.p. $150 \sim 152$ ${ }^{\circ} \mathrm{C} ;{ }^{1} \mathrm{H}$ NMR $\left(\mathrm{CDCl}_{3}, 300 \mathrm{MHz}\right) \delta: 9.87(\mathrm{~s}, 1 \mathrm{H}), 8.57$ (d,
$J=4.90 \mathrm{~Hz}, 4 \mathrm{H}), 7.71 \sim 7.75(\mathrm{~m}, 2 \mathrm{H}), 7.50(\mathrm{~d}, J=8.40 \mathrm{~Hz}$, $4 \mathrm{H}), 7.36(\mathrm{~d}, J=4.85 \mathrm{~Hz}, 4 \mathrm{H}), 7.14 \sim 7.18(\mathrm{~m}, 4 \mathrm{H}), 7.05 \sim$ $7.08(\mathrm{~m}, 2 \mathrm{H}), 6.91 \sim 6.99(\mathrm{~m}, 4 \mathrm{H})$; IR (KBr) v: 3058, 2837, $1690,1628,1603,1572,1479,952 \mathrm{~cm}^{-1}$.

$3.2 .24-[N, N-$ 二 $(((N-$ 一八烷基 $)$ 吡啶碘盐 -4 -乙烯基 $)$ 苯基)氨基]苯甲醛(TPAPyS)的合成

取 4- $\{N, N$-二[4-(4-吡啶乙烯基)苯基]氨基 $\}$ 苯甲醛 $(0.600 \mathrm{~g}, 1.25 \mathrm{mmol})$ 、碘代十八烷 $(1.901 \mathrm{~g}, 5.00 \mathrm{mmol})$ 、 丙酠 $30 \mathrm{~mL}$ 于 $50 \mathrm{~mL}$ 单口瓶中, 升温至 $60{ }^{\circ} \mathrm{C}$ 反应 $48 \mathrm{~h}$, 反应结束后将溶剂旋干，得到的固体用甲醇重结晶，得 到红色固体 $0.54 \mathrm{~g}$, 产率 $43.8 \%$. m.p. $173 \sim 175{ }^{\circ} \mathrm{C} ;{ }^{1} \mathrm{H}$ NMR $\left(\mathrm{CDCl}_{3}, 300 \mathrm{MHz}\right) \delta: 9.90(\mathrm{~s}, 1 \mathrm{H}), 8.92 \sim 8.94(\mathrm{~m}$, $4 \mathrm{H}), 8.25 \sim 8.23(\mathrm{~m}, 4 \mathrm{H}), 7.87 \sim 7.84(\mathrm{~m}, 2 \mathrm{H}), 7.68 \sim 7.75$ $(\mathrm{m}, 4 \mathrm{H}), 7.10 \sim 7.07(\mathrm{~m}, 6 \mathrm{H}), 6.92 \sim 7.00(\mathrm{~m}, 4 \mathrm{H}), 4.68 \sim$ $4.70(\mathrm{~m}, 4 \mathrm{H}), 1.63 \sim 2.01(\mathrm{~m}, 4 \mathrm{H}), 1.26 \sim 1.34(\mathrm{~m}, 60 \mathrm{H})$, $0.89(\mathrm{t}, J=6.00 \mathrm{~Hz}, 6 \mathrm{H}) ;{ }^{13} \mathrm{C} \mathrm{NMR}\left(\mathrm{CDCl}_{3}, 300 \mathrm{MHz}\right) \delta$ : $191.75,154.92,152.61,149.05,144.95,142.34,132.65$, $132.41,131.62,126.27,125.71,124.24,122.98,62.24$ $33.11,32.83$, 30.91, 30.77, 30.31, 27.34, 23.87, 15.31; IR (KBr) v: 3029, 1693, 1653, 1584, 1502, 1466, 1157, 966 $\mathrm{cm}^{-1}$; HRMS-MALDI-TOF calcd for $\mathrm{C}_{69} \mathrm{H}_{99} \mathrm{~N}_{3} \mathrm{I}_{2} \mathrm{O}[\mathrm{M}+$ $\mathrm{H}-2 \mathrm{I}]^{3+}$ 986.7845, found 986.7912 .

\section{References}

[1] Kulinich, A. V.; Ishchenko, A. A. Chem. Rev. 2009, 78, 141.

[2] Qi, X.; Jun, E. J.; Xu, Li.; Kim, S.-J. J. Org. Chem. 2006, 71, 2881.

[3] Wu, F.-Y.; Xie, F.-Y.; Wu, Y.-M.; Hong, J.-I. J. Fluoresc. 2008, 18, 175 .

[4] He, Q. W.; Miller, E. W.; Wong, A. P.; Chang, C. J. Am. Chem. Soc. 2006, 128, 9316 .

[5] Tang, B.; Huang, H.; Xu, K. H.; Tong, L. L.; Yang, G. W.; Liu, X.; An, L. G. Chem. Commun. 2006, 3609.

[6] Hu, C.; Sun, W.; Cao, J. F.; Gao, P.; Wang, J. Y. Org. Lett. 2013, 15, 4022.

[7] Zhang, X. J.; Ren, X. S.; Xu, Q. H.; Chen, Z. K. Org. Lett. 2009, $11,1257$.

[8] Zhang, C. J.; Li, L.; Chen, G. Y. J.; Xu, Q. H.; Yao, S. Q. Org. Lett. 2011, 13, 4160 .

[9] Koide, Y.; Urano, Y.; Hanaoka, K.; Terai, T.; Nagano, T. J. Am. Chem. Soc. 2011, 133, 5680.

[10] Abnet, C. C.; Wang, Z. M.; Song, X.; Hu, N.; Zhou, F.-Y. Human Mol. Gen. 2012, 21, 9

[11] Liu, X.; Sun, Y. M.; Zhang, Y. H.; Zhao, N. J. Fluoresc. 2011, 21, 497.

[12] Chen, Y. G.; Guo, W. H.; Ye, Z. Q.; Wang, G. L.; Yuan, J. L. Chem. Commun. 2011, 47, 6266.

[13] Kundu, K.; Knight, S. F.; Willet, N.; Lee, S. M.; Taylor, W. R.; Murthy, N. Angew. Chem., Int. Ed. 2009, 48, 299.

[14] Naama, K.-L.; Ehud, S.; Liora, O.; Moshe, P.; Ronit, S.-F.; Doron, S. J. Am. Chem. Soc. 2011, 133, 10960.

[15] Kiyose, K.; Hanaoka, K.; Oushiki, D.; Nakamura, T.; Kajimura, M. J. Am. Chem. Soc. 2010, 132, 15846.

[16] Jiang, G. X.; Susha, A. S.; Lutich, A. A.; Stefani, F. D. J. Am. Chem. 
Soc. 2009, 3, 4127.

[17] Chandran, S. S.; Dickson, K. A.; Raines, R. T. J. Am. Chem. Soc. 2005, 127, 1652.

[18] Jyotirmayee, M.; Nilotpal, B. J. Am. Chem. Soc. 2013, 135, 367.

[19] Lee, H.; Berezin, M. Y.; Guo, K.; Kao, J.; Achilefu, S. Org. Lett. 2009, 11, 29.

[20] Almutairi, A.; Guillaudeu, S. J.; Berezin, M. Y.; Achilefu, S. J. Am. Chem. Soc. 2008, 130, 444.

[21] Xu, C.-X.; Xu, H.-G.; Meng, R.-P.; Feng, Y.-X.; Zhang, J.-Y.; Cui, Y.-P. J. Chin. Luminesc 2006, 27, 679 (in Chinese). (徐春祥, 徐洪光, 发光学报, 2006, 27, 679.)

[22] Jin, K.-J.; Chen, X.-J.; Liu, Y.; Qin, A,-J.; Tang, B.-Z. Acta Polym. Sinica 2011, 27, 1079 (in Chinese). (金科佳，陈秀娟，刘一，高分子学报, 2011, 27, 679.)

[23] Elacqua, E.; Bucar, D.-K. Org. Lett. 2009, 11, 5106.

[24] Blaise, D.; Guillaume, B.; Elodie, F.-P.; Florence, M.-B. J. Am. Chem. Soc. 2013, 135, 12697.

[25] Moineau, J.; Pozzi, G.; Quici, S.; Sinou, D. Tetrahedron Lett. 1999, $40,7683$.

[26] Yuan, C.-X.; Tao, X.-T.; Ren, Y.; Li, Y. J. Phys. Chem. C 2007, 111, 12811.

[27] Qin, P.; Liu, R.; Pan, X.; Fang, X.; Mou, Y. J. Agric. Food Chem. 2010, 58,5561 .

[28] Bhattacharya, B.; Nakka, S.; Guruprasad, L.; Samanta, A. J. Phys. Chem. B 2009, 113, 2143.

[29] Guharay, J.; Sengupta, B.; Sengupta, P. K. Proteins 2001, 43, 75.

[30] Shu, Y.; Liu, M. L.; Chen, S.; Chen, X. W. J. Phys. Chem. B 2011, $115,12306$.
[31] Ghosh, S.; Jana, S.; Guchhait, N. J. Phys. Chem. B 2012, 116, 1155.

[32] Jiang, G.-Y.; Lei, W.-H.; Zhou, Q.-X. Photochem. Photobiol. Sci. 2012, 11, 715 .

[33] Maurice, M. S.; Bearne, S. L. Biochemistry 2004, 43, 2524.

[34] Shen, J.-B.; Tong, B.; Shi, J. B.; Sun, S. Acta Polym. Sinica 2010, 765 (in Chinese).

(申进波, 佟斌, 石建兵, 孙书, 高分子学报, 2010, 765.)

[35] Yang, Z.-Y.; Y, T.; Chen, M.-N.; Zhang, X.-Q. Acta Polym. Sin. 2009, 560 (in Chinese).

(杨志涌, 于涛, 陈美娜, 张锡奇, 高分子学报, 2009, 560.)

[36] Hong, Y. N.; Feng, C.; Yu, Y.; Liu, J.; Tang, B.-Z. Anal. Chem. 2010, 82, 7035 .

[37] Luo, Y.; Huang, X.-C. Acta Chim. Sinica 2012, 70, 1295 (in Chinese). (罗懿, 黄一纯, 化学学报, 2012, 70, 7035.)

[38] Peng, G.; Liu, B.-L.; Zhao, C. X.; Jiang, Z. W. J. Graduate School Chin. Academy Sci. 2011, 28, 12.

[39] Chen, Y. H.; Yang, J. T. Biochemistry 1972, 11, 4120.

[40] Gao, H.; Lei, L. D.; Liu, J. Q. J. Photochem. Photobiol. A 2004, 167, 213..

[41] Lin, W. Y.; Long, L. L.; Yuan, L.; Cao, Z. M.; Chen, B. B. Org. Lett. 2008, 10, 5577.

[42] Madhu, S.; Gonnade, R.; Ravikanth, M. J. Org. Chem. 2013, 78, 5056.

[43] Luo, M. L.; Ying, Q. Chin. J. Org. Chem. 2012, 32, 1958 (in Chinese).

(罗蔓利, 钱鹰, 有机化学, 2012, 32, 1958.)

(Qin, X.) 